\title{
e-ISSN $1983-0572$ \\ Fauna de Coleoptera Associada a Carcaças de Coelhos Expostas em uma Área Urbana no Sul do Brasil
}

\author{
Richard Cordeiro da Silva ${ }^{1} \&$ Wellington Emanuel dos Santos ${ }^{2} \bowtie$
}

1. Universidade Estadual do Norte do Paraná, CCHE/CJ, e-mail: richardcordeiro84@hotmail.com. 2. Programa de Pós-Graduação em Ciências Biológicas (Zoologia). Laboratório de Entomologia, Departamento de Sistemática e Ecologia, Universidade Federal da Paraíba, e-mail: well-bio@hotmail.com. (Autor para correspondência ${ }^{\bowtie}$ ).

\section{EntomoBrasilis 5 (3): 185-189 (2012)}

\begin{abstract}
Resumo. Estudo da fauna de Coleoptera associada a carcaças de coelhos, Oryctolagus cuniculus Linnaeus (Lagomorpha, Leporidae), durante o verão de 2010 e o inverno de 2011, em uma área urbana do norte do Paraná, sul do Brasil, é apresentado. Para captura dos coleópteros foram utilizadas armadilhas do tipo bandeja, pitfall e Shannon modificada. Foram coletados 236 coleópteros pertencentes a 12 famílias e pelo menos 25 espécies. Staphylinidae $(52,5 \%)$ e Histeridae $(26,7 \%)$ foram as famílias mais abundantes em ambas as estações, representadas principalmente por Aleochara bonariensis Lynch e Euspilotus "grupo" azureus sp., respectivamente. Entre as estações, tanto a decomposição das carcaças quanto a sucessão da coleopterofauna de interesse forense ocorreram de forma distinta, devido à variação expressiva dos fatores abióticos. Durante o inverno, a maioria dos indivíduos de Euspilotus "grupo" azureus sp. (96,8\%) foi capturada no estágio de Putrefação Escura, revelando uma associação a esse estágio. Apesar da sazonalidade do ambiente, não houve diferença significativa da abundância dos coleópteros mais coletados, nem da coleopterofauna em geral, entre as estações.
\end{abstract}

Palavras-Chave: Besouros; Entomologia Forense; Histeridae; Staphylinidae.

\section{Coleoptera Fauna Associated with Rabbit Carcasses Exposed in an Urban Area in Southern Brazil}

Abstract. Study of Coleoptera fauna associated with rabbit carcasses, Oryctolagus cuniculus Linnaeus (Lagomorpha, Leporidae), during the summer of 2010 and winter of 2011, is present at an urban area of Northern Paraná State, Southern Brazil. To capture the beetles were used pan, pitfall and modified Shannon traps. We collected 236 beetles belonging to 12 families and at least 25 species. Staphylinidae (52.5\%) and Histeridae (26.7\%) were the most abundant families in both seasons represented mainly by Aleochara bonariensis Lynch and Euspilotus "group" azureus sp., respectively. Among the seasons, both the decomposition of the carcasses and the succession of coleopterofauna of forensic interest occurred differently due to significant variation of abiotic factors. During the winter, most individuals of Euspilotus "group" azureus sp. (96.8\%) was captured at the stage of Black Putrefaction, showing an association at this stage. Despite the environment seasonality, there wasn't significant difference of the abundance among seasons by main beetles neither by coleopterofauna.

Keywords: Beetles; Forensic Entomology; Histeridae; Staphylinidae.

C om a ocorrência da morte o corpo começa a sofrer diversas mudanças ao longo da decomposição. Esse processo sofre influência de diversos fatores externos, tais como: temperatura, umidade, precipitação e, peculiarmente, entomofauna decompositora. Essa fauna é representada principalmente por moscas (Diptera), besouros (Coleoptera) e formigas (Hymenoptera) (BENECKE 2001; CAMPoBASso et al. 2001).

Quando esses insetos encontram um cadáver inicia-se uma sucessão, pois condições e características que atraem determinados grupos de insetos são oferecidas gradativamente no decorrer da decomposição (Bornemissza 1957; Oliveira-Costa 2011). Dessa forma, baseado no período em que os insetos habitam a carcaça é possível determinar certas circunstâncias nas quais a morte ocorreu e, principalmente, estimar o Intervalo Pós-Morte (IPM) (KeH 1985; CATTS \& GoFf 1992).

A ordem Coleoptera é considerada a segunda de maior interesse forense, depois de Diptera. Em associação com as carcaças existem poucas espécies efetivamente necrófagas, sendo a maioria predadora, porém ambas as categorias podem ser informativas (Goff 1991). De acordo com Kulshresta \& SATpathy (2001), quando são encontrados esqueletos de humanos na fase seca, os coleópteros tornam-se a principal evidência entomológica para determinação do Intervalo pós-morte (IPM), baseado principalmente no padrão de sucessão. A aplicabilidade forense dos coleópteros também foi demonstrada por GARCíA-RoJo et al. (2009) que, a partir das espécies presentes, determinaram em aproximadamente um ano o IPM de dois cadáveres encontrados no interior de uma residência em avançado estágio de decomposição.

O Brasil possui a maior biodiversidade do mundo e cada bioma apresenta fauna e condições regionais particulares, as quais podem, ainda, variar entre as estações do ano (PuJoL-Luz et al. 2008; ANDERSON 2001). Tais fatos refletem diretamente sobre a entomofauna cadavérica. Assim, estudos regionais são essenciais para a utilização da Entomologia Forense.

Visto que os levantamentos com foco na fauna de Coleoptera são escassos no Brasil (Luederwaldt 1911; PessôA \& LANE 1941; Mise et al. 2007, 2008, 2010) e que o conhecimento sobre a sucessão, distribuição geográfica e sazonalidade desses insetos são importantes para a Entomologia Forense (PuJoL-Luz et al. 2008; ANDERSON 2001), o presente trabalho teve como objetivo estudar a fauna de Coleoptera associada a carcaças de coelhos, Oryctolagus cuniculus Linnaeus (Lagomorpha, Leporidae), 
durante o verão e o inverno, expostas em uma área urbana do estado do Paraná, Sul do Brasil. Adicionalmente, notas sobre o processo de decomposição e atividade de dípteros foram realizadas.

\section{MATERIAL E MÉTODOS}

O experimento foi realizado em uma área urbana do município de Ribeirão do Pinhal, região Norte do estado do Paraná, Sul do Brasil. Mais precisamente no quintal de uma residência situada no bairro Jardim Bandeirantes, localizado na extremidade leste da cidade $\left(23^{\circ} 25^{\prime} \mathrm{S}, 50^{\circ} 21^{\prime} \mathrm{W}\right)$. As coletas foram realizadas entre 20 de dezembro de 2010 e 04 de janeiro de 2011 (verão) e entre 04 e 23 de julho de 2011 (inverno). Em cada estação como substrato foi utilizada uma carcaça de coelho, $O$. cuniculus, macho com peso médio de $1,5 \mathrm{~kg}$, sacrificada por deslocamento cervical.

As carcaças foram expostas em uma gaiola conforme modelo proposto por Monteiro-Filho \& Penereiro (1987). Confeccionouse uma armação de madeira $(50 \times 50 \times 50 \mathrm{~cm})$ revestida com tela de arame (malha de $1,5 \times 1,5 \mathrm{~cm}$ ). Na parte inferior foi colocada uma bandeja (70 × $70 \times 15 \mathrm{~cm})$, enterrada ao nível do solo, contendo areia e maravalhas para captura ativa dos coleópteros abaixo da carcaça (SANTOS 2012). Também foram colocadas quatro armadilhas pitfall ao redor da gaiola, contendo água e gotas de detergente, para captura passiva. Além disso, a gaiola foi coberta por uma armadilha Shannon modificada $(1,5 \times 1,1 \mathrm{~m})$ com um tubo coletor, contendo álcool $70 \%$, acoplada na parte superior para capturar os coleópteros voadores (Mise et al. 2007).

Para aferição da temperatura e umidade relativa foi instalado um termo-higrômetro digital no local. Os dados de precipitação foram obtidos através da Estação Agrometeorológica da Universidade Estadual do Norte do Paraná.

As carcaças foram vistoriadas diariamente por duas horas, tempo este dividido entre a coleta nas armadilhas e observação dos estágios de decomposição, que seguiram a classificação de BorNEMISSZA (1957). As coletas foram finalizadas quando as carcaças atingiram o final do estágio Seco de decomposição, restando apenas pelos e ossos, ocasião em que não mais se observaram coleópteros, nem larvas de dípteros.

O material coletado foi identificado com base nas seguintes chaves de identificação: REICHARDT (1977), MAZUR (2001), ARNETT et al. (2002), NAVARRETE-HEREDia et al. (2002), Almeida \& Mise (2009) e VAZ-DE-MELLo et al. (2011), além da ajuda do especialista Dr. Kleber Makoto Mise, da Universidade Federal do Paraná. Para determinar as famílias de importância forense adotou-se a classificação de Sмiтн (1986), ao passo que a classificação dos hábitos alimentares seguiu o proposto por CATTS \& GoFf (1992).

Os coleópteros foram depositados na Coleção Entomológica do Laboratório de Zoologia da Universidade Estadual do Norte do Paraná - UENP, CCHE/CJ.

\section{RESULTADOS}

Foram coletados 236 coleópteros pertencentes a 12 famílias, sete de interesse forense e cinco acidentais, e pelo menos 25 espécies (Tabela 1). A família Staphylinidae foi a mais abundante, com 124 espécimes, representando 52,5\% do total de coleópteros capturados. Entre os representantes dessa família, a espécie mais coletada foi Aleochara bonariensis Lynch, em ambas as estações de realização do experimento. A família Histeridae foi a segunda em abundância, com 63 indivíduos (26,7\%), representada, principalmente, por Euspilotus "grupo" azureus sp. em ambas as estações. Dentre os métodos de coleta adotados a coleta ativa realizada na bandeja foi o que obteve maior abundância e riqueza de espécies, seguido pelas pitfalls e Shannon modificada.
Com relação ao processo de decomposição, nas duas estações foram observados quatro dos cinco estágios propostos por Bornemissza (1957). O de estágio de Fermentação não foi observado. Ao longo desses estágios, entre as estações, tanto a decomposição das carcaças quanto a sucessão da coleopterofauna de interesse forense ocorreram de forma distinta.

O estágio de Decomposição Inicial durante o verão teve a duração de apenas um dia e nele não foram observados coleópteros, apenas muitos dípteros que chegaram à carcaça após poucos minutos de exposição em campo. No inverno, esse estágio durou seis dias e durante este tempo não foram observados coleópteros, nem dípteros sobrevoando a carcaça.

O estágio de Putrefação foi caracterizado pelo inchaço da carcaça devido ao acúmulo de gases internos. Durante o verão esse estágio durou apenas um dia, foi capturado um exemplar de Lagria villosa Fabricius (Tenebrionidae) e observada grande quantidade de dípteros sobrevoando a carcaça. No inverno, o estágio durou quatro dias. Nesse período houve a captura de um exemplar de $A$. bonariensis e apareceram os primeiros dípteros adultos sobre a carcaça.

A Putrefação Escura foi caracterizada por um forte odor de putrefação devido ao rompimento da carcaça, permitindo o escape dos gases internos. Grande quantidade de larvas de dípteros foi observada, principalmente nas regiões da cabeça e ânus, as quais permaneceram na carcaça até o estágio Seco de decomposição. Na coleta do verão, esse estágio durou apenas um dia, assim como os anteriores, e não havia coleópteros na carcaça. Durante o inverno, o estágio de Putrefação Escura durou quatro dias e nele foi capturado o maior número de coleópteros da estação. Incluindo a maioria dos indivíduos de Euspilotus "grupo" azureus sp. (96,8\%) e de $A$. bonariensis (62,1\%), revelando uma maior associação desses coleópteros a esse estágio durante o inverno.

Nos dias iniciais do estágio Seco as carcaças apresentavam-se secas por fora, mas com algumas partes internas com consistência cremosa e com um odor de putrefação mais ameno. Ao final do estágio, as carcaças apresentavam-se totalmente secas, restando apenas pelos e ossos. Durante o verão o estágio Seco foi observado a partir do quarto dia de exposição da carcaça e teve uma duração de treze dias. Nesse período, ocorreu a colonização de quase todos os coleópteros de interesse forense. Já no inverno, estágio Seco de decomposição só foi observado a partir do $15^{\circ}$ dia de exposição da carcaça e teve duração de seis dias, nos quais se verificou um declínio gradual dos coleópteros capturados. Aparentemente, em ambas as estações, a sucessão da coleopterofauna, principalmente dos predadores/parasitas, seguiu os dias da decomposição, acompanhados do desenvolvimento de suas presas, e não os estágios de decomposição da carcaça.

A variação da duração dos estágios de decomposição entre as estações está provavelmente ligada à variação expressiva dos fatores abióticos observada. Durante o verão foi registrada temperatura média de $26,1 \pm 1,5^{\circ} \mathrm{C}$, com máxima de $34,4^{\circ} \mathrm{C}$ e mínima de $16,3^{\circ} \mathrm{C}$, umidade relativa média de $56,0 \pm 10,6 \%$, com máxima de 77,0\% e mínima de $34,5 \%$, e precipitação acumulada de $33,2 \mathrm{~mm}$. No inverno foi registrada temperatura média de $20,4 \pm 3,2^{\circ} \mathrm{C}$, com máxima de $31,8^{\circ} \mathrm{C}$ e mínima de $8,0^{\circ} \mathrm{C}$, umidade relativa média de 53,0 $\pm 14,5 \%$, com máxima de 99,0\% e mínima de 20,0\%, e precipitação acumulada de 24,5 mm. Esses fatores não provocaram diferença significativa na abundância da coleopterofauna entre as estações, mesmo com uma maior riqueza de espécies observada no verão. Dentre os coleópteros mais coletados, também não foi observada preferência por nenhuma estação. Apenas uma pequena maioria dos indivíduos de $A$. bonariensis (57,9\%) e Euspilotus "grupo" azureus sp. $(53,4 \%)$ foi coletada no inverno, provavelmente em função das peculiaridades biológicas, ainda desconhecidas, dessas espécies. 
Tabela 1. Abundância dos coleópteros em cada fase da decomposição, durante o verão e o inverno, e seus respectivos métodos de coleta. DI: Decomposição Inicial; P: Putrefação; PE: Putrefação Escura; S: Seco; D: Dias da decomposição.

\begin{tabular}{|c|c|c|c|c|c|c|c|c|c|c|c|c|c|c|}
\hline \multirow{3}{*}{ 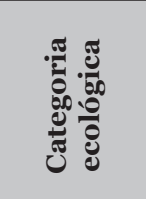 } & \multirow{3}{*}{ Família } & \multirow{3}{*}{ Espécies } & \multicolumn{4}{|c|}{ Verão } & \multicolumn{4}{|c|}{ Inverno } & \multicolumn{3}{|c|}{ Método de coleta } & \multirow{3}{*}{ సี } \\
\hline & & & DI & $\mathbf{P}$ & PE & $\mathbf{S}$ & DI & $\mathbf{P}$ & $\mathbf{P E}$ & $\mathbf{S}$ & & & $\tilde{\xi}$ & \\
\hline & & & D 1 & D 2 & D 3 & $\underset{4-16}{D}$ & D 1-6 & $\underset{7-10}{D}$ & $\stackrel{D}{D}$ & $\begin{array}{c}D \\
15^{-20}\end{array}$ & $\stackrel{\sum}{2}$ & 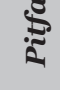 & $\frac{\tilde{\Xi}}{\tilde{\Xi}}$ & \\
\hline Necrófago & Cleridae & Necrobia rufipes & - & - & - & - & - & - & 1 & - & 1 & - & - & 1 \\
\hline \multirow{12}{*}{ 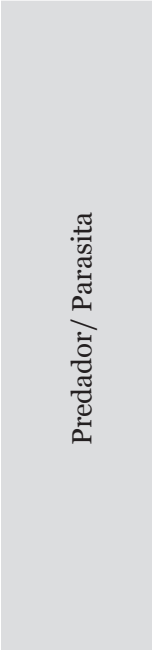 } & \multirow{3}{*}{ Carabidae } & Bembidiini sp. & - & - & - & 1 & - & - & - & - & 1 & - & - & 1 \\
\hline & & Omophronini sp. & - & - & - & - & - & - & 1 & - & - & 1 & - & 1 \\
\hline & & Pogonini sp. & - & - & - & 1 & - & - & - & - & - & 1 & - & 1 \\
\hline & \multirow{3}{*}{ Histeridae } & $\begin{array}{l}\text { Euspilotus "grupo" } \\
\text { azureus sp. }\end{array}$ & - & - & - & 27 & - & - & 30 & 1 & 54 & 4 & - & 58 \\
\hline & & Hister sp. & - & - & - & 3 & - & - & - & - & 3 & - & - & 3 \\
\hline & & Phelister sp. & - & - & - & 2 & - & - & - & - & 2 & - & - & 2 \\
\hline & \multirow{6}{*}{ Staphylinidae } & Aleochara bonariensis & - & - & - & 48 & - & 1 & 41 & 24 & 107 & 4 & 3 & 114 \\
\hline & & Belonuchus sp. & - & - & - & - & - & - & 1 & - & 1 & - & - & 1 \\
\hline & & $\begin{array}{l}\text { Neohypnos aff. } \\
\text { attenuates }\end{array}$ & - & - & - & 5 & - & - & - & - & 5 & - & - & 5 \\
\hline & & Osorius sp. & - & - & - & 2 & - & - & - & - & 1 & 1 & - & 2 \\
\hline & & Piestus sp. & - & - & - & 1 & - & - & - & - & 1 & - & - & 1 \\
\hline & & Xanthopygus sp. & - & - & - & 1 & - & - & - & - & 1 & - & - & 1 \\
\hline \multirow{7}{*}{ Onívoro } & \multirow{3}{*}{ Nitidulidae } & Carpophilus sp. & - & - & - & 3 & - & - & - & - & 3 & - & - & 3 \\
\hline & & Colopterus sp. & - & - & - & - & - & - & - & 1 & - & - & 1 & 1 \\
\hline & & Stelidota sp. & - & - & - & 1 & - & - & - & - & 1 & - & - & 1 \\
\hline & \multirow{3}{*}{ Scarabaeidae } & Ataenius sp. & - & - & - & 11 & - & - & 3 & 1 & 14 & 1 & - & 15 \\
\hline & & Canthon sp. & - & - & - & 5 & - & - & - & - & 3 & 2 & - & 5 \\
\hline & & Canthidium sp. & - & - & - & 1 & - & - & - & - & - & 1 & - & 1 \\
\hline & Tenebrionidae & Lagria vilosa & - & 1 & - & 3 & - & - & - & - & 2 & 2 & - & 4 \\
\hline \multirow{5}{*}{ Acidental } & Bostrichidae & Bostrichidae sp. & - & - & - & 1 & - & - & - & - & 1 & - & - & 1 \\
\hline & Cerambycidae & Cerambycidae spp. & - & - & - & 1 & 1 & - & - & - & - & 1 & 1 & 2 \\
\hline & Chrysomelidae & Chrysomelidae spp. & - & - & - & 4 & - & - & - & 1 & 2 & 1 & 2 & 5 \\
\hline & Curculionidae & Curculionidae spp. & - & - & - & 2 & - & 3 & - & - & 1 & 1 & 3 & 5 \\
\hline & Meloidae & Meloidae sp. & - & 1 & - & 1 & - & - & - & - & 1 & - & 1 & 2 \\
\hline Total & & & - & 2 & - & 124 & 1 & 4 & 77 & 28 & 205 & 20 & 11 & 236 \\
\hline
\end{tabular}

\section{DISCUSSÃO}

Dentre as famílias coletadas, Carabidae, Cleridae, Histeridae, Nitidulidae, Scarabaidae, Staphylinidae e Tenebrionidae possuem interesse forense (Sмітн 1986), por apresentarem hábito alimentar necrófago, predador/parasita ou onívoro (CATTs \& Goff 1992). A grande representatividade das famílias Staphylinidae e Histeridae está ligada, provavelmente, ao fato de seus representantes serem predadores de larvas de dípteros, recurso altamente disponível nas carcaças, e as suas tendências de agregação sobre o recurso (CATTS \& GoFf 1992; Moura 2004; Mise et al. 2007).

Em experimentos realizados na cidade de Curitiba, em um fragmento de floresta ombrófila mista, Mise et al. (2007), utilizando carcaças de suínos, também relataram a família Staphylinidae como a de maior abundância em todos os estágios de decomposição, seguido por Histeridae no estágio de Putrefação e Putrefação Escura e por Silphidae no Estágio de Fermentação. Ainda no trabalho de Mise et al. (2007), Aleochara (Staphylinidae) foi o gênero mais coletado, assim como no presente trabalho. A maior abundância de $A$. bonariensis constatada em relação às outras espécies pode ser explicada, provavelmente, pelo fato de indivíduos do gênero quando adultos predarem ovos e larvas de dípteros e durante a fase larval serem parasitas de pupários (Mise et al., 2007).
Quanto a família Histeridae, Souza \& LiNHAREs (1997) coletaram as espécies Euspilotus azureus (Sahlberg), Euspilotus sp. e Omalodes sp. durante os estágios de Putrefação, Putrefação Escura e Fermentação, em carcaças de suínos, em Campinas. Mise et al. (2007) coletaram 519 exemplares da família durante os seguintes estágios de decomposição: Fresco, Putrefação, Putrefação Escura e Fermentação. A família foi representada, principalmente, por Euspilotus "grupo" azureus sp., seguida por Hister sp. e Phelister sp., além de Euspilotus sp., fato que também foi registrado no presente trabalho, levantando a possibilidade de haver semelhança na fauna de histerídeos associados a carcaças no estado do Paraná.

A expressividade da coleta ativa na bandeja deve-se ao posicionamento da carcaça em contato direto com a armadilha, a qual simula o solo e, assim, concentra as larvas de dípteros sob a carcaça (SANTOS 2012). Utilizando metodologia semelhante, porém com bandeja não enterrada ao nível do solo, Mise et al. (2007) coletaram 46,3\% dos coleópteros nessa armadilha e registraram maior riqueza na Shannon modificada. O intuito de comparar os métodos de coleta é destacar suas diferenças, enfatizando que são complementares umas às outras. Assim, uma metodologia de coleta não deve abrir mão de usar diferentes formas de captura.

Baixas temperaturas associadas à baixa umidade relativa do ar 
são responsáveis por um processo de conservação da carcaça (McAlpine 1965; Monteiro-Filho \& Penereiro 1987). Enquanto que altas temperaturas e elevada umidade aceleram a decomposição, já que tais fatores atuam sobre a ação bacteriana, e atraem maior riqueza e abundância de insetos para os cadáveres (CAMPOBASSO et al. 2001). Tais constatações corroboraram o observado: uma rápida decomposição no verão, com maior riqueza de espécies, e uma decomposição mais lenta durante o inverno, com poucas espécies presentes.

O presente trabalho e os de Mise et al. (2007, 2008), mesmo sendo realizados no estado do Paraná, apresentaram diferenças quanto a composição taxonômica e abundância da coleopterofauna. No presente estudo não foram constatadas algumas famílias de importância forense citadas pelos autores, como as famílias Dermestidae, Silphidae e Trogidae, amplamente coletas em carcaças no Brasil (LuEderwaldT 1911; SouZA \& LinHARES 1997; Ururahy-Rodrigues et al. 2010; Rosa et al. 2011). Por outro lado, Ataenius sp. (Scarabaeidae), Osorius sp., Piestus sp. e Xanthopygus sp. (Staphylinidae), presentes neste trabalho não foram coletadas por Mise et al. $(2007,2008)$.

Mesmo tendo sido utilizados modelos animais diferentes, estas diferenças constatadas remetem a diferença na área de realização dos estudos: o presente trabalho em área urbana e os trabalhos de Mise et al. (2007, 2008) em fragmento de floresta. Assinalando, assim, a necessidade de estudos em diferentes áreas e regiões mesmo tratando-se do mesmo estado para um estabelecimento fidedigno da Entomologia Forense no Brasil. Adicionalmente, estudos futuros sobre biologia das espécies mais abundantes, A. bonariensis e Euspilotus "grupo" azureus sp., se fazem necessários para um franco entendimento do papel que esses coleópteros desempenham nas carcaças.

\section{AGRADECIMENTOS}

Os autores agradecem ao Dr. Kleber Makoto Mise, da Universidade Federal do Paraná, pelo auxílio nas identificações, e aos revisores anônimos pelas contribuições ao manuscrito.

\section{REFERÊNCIAS}

Almeida, L.M. \& K.M. Mise, 2009. Diagnosis and key of the main families and species of South American Coleoptera of forensic importance. Revista Brasileira de Entomologia, 53: 227-244.

Anderson, G.S., 2001. Insect succession on carrion and its relationship to determining time of death, p. 143-175. In: Byrd, J.H. \& J.L. Castner (Eds.). Forensic Entomology: the utility of arthropods in legal investigations. Boca Raton, CRC Press, 418p.

Arnett, R.H., M.C. Thomas, P.E. Skelley \& J.H. Frank (Eds.), 2002. American Beetles, Volume 2: Polyphaga: Scarabaeoidea through Curculionoidea. Boca Raton, CRC Press, 861p.

Benecke, M., 2001. A brief history of forensic entomology. Forensic Science International, 120: 2-14.

Bornemissza, G.F., 1957. An analysis of arthropod sucession in corion and the effect of its decomposition on the soil fauna. Australian Journal of Zoology, 5: 1-12.

Campobasso, C.P., G. Vella \& F. Introna, 2001. Factors affecting decomposition and Diptera colonization. Forensic Science International, 120: 18-27.

Catts, E.P. \& M.L. Goff, 1992. Forensic entomolgy in criminal investigations. Annual Review of Entomology, 27: 253-272.

García-Rojo, A.M., L. Honorato, M. González \& A. Téllez, 2009. Determinación del intervalo postmortem mediante el estudio de la sucesión de insectos en dos cadáveres hallados em el interior de una finca rústica en Madrid. Cuadernos de Medicina Forense, 15: 137-145.

Goff, M.L., 1991. Comparison of insect species associated with decomposing remains recovered inside dwellings and outdoors of the island of Oahu, Hawaii. Journal of Forensic Science, 36: 748-753.
Keh, B., 1985. Scope and applications of Forensic Entomology. Annual of Review Entomology, 30: 137-154.

Kulshrestha, P. \& D.K. Satpathy, 2001. Use of beetles in forensic entomology. Forensic Science International, 120: 15-17.

Luederwaldt, H., 1911. Os insetos necrófagos paulistas. Revista do Museu Paulista, 8: 414-433.

Mazur, S., 2001. Review of the Histeridae (Coleoptera) of México. Dugesiana, 8: 17-66.

McAlpine, J.F., 1965. Insects and related terrestrial invertebrates of Ellef Ringnes Island. Journal of the Arctic Institute of North America, 18: 73-103.

Mise, K.M., A.S.B. Souza, C.M. Campos, R.L.F. Keppler \& L.M. Almeida, 2010. Coleoptera associated with pig carcass exposed in a forest reserve, Manaus, Amazonas, Brazil. Biota Neotroprica, 10: 320-324.

Mise, K.M., C.B.C. Martins, E.L. Köb \& L.M. Almeida, 2008. Longer decomposition process and the influence on Coleoptera fauna associated with carcasses. Brazilian Journal of Biology, 68: 907-908.

Mise, K.M., L.M. Almeida \& M.O. Moura, 2007. Levantamento da fauna de Coleoptera que habita a carcaça de Sus scrofa L., em Curitiba, Paraná. Revista Brasileira de Entomologia, 51: 358-368.

Monteiro-Filho, E.L.A. \& J.L. Penereiro, 1987. Estudo de decomposição e sucessão sobre uma carcaça animal numa área do estado de São Paulo. Revista Brasileira de Biologia, 47: 289-295.

Moura, M.O., 2004. Variação espacial como mecanismo promotor da coexistência em comunidade de insetos necrófagos. Revista Brasileira de Zoologia, 21: 409-419.

Navarrete-Heredia, J.L., A.F. Newton, M.K. Thayer, J.S. Ashe \& D.S. Handler, 2002. Guía ilustrada para los géneros de Staphylinidae (Coleoptera) de México. Illustrated guide to the genera of Staphylinidae (Coleoptera) of México. México, Universidad de Guadalajara y CONABIO, 401p.

Oliveira-Costa, J., 2011. Entomologia forense: quando os insetos são vestígios, $3^{\text {a }}$ Ed. Campinas, Millennium, 520p.

Pessôa, S.B. \& F. Lane, 1941. Coleópteros necrophagos de interesse médico-legal. Ensaio monográfico sobre a família Scarabaeidae de São Paulo e regiões vizinhas. Arquivos de Zoologia do Estado de São Paulo, 2: 389-504.

Pujol-Luz, J.R., L.C. Arantes \& R. Constantino, 2008. Cem anos da Entomologia Forense no Brasil (1908-2008). Revista Brasileira de Entomologia, 52: 485-492.

Reichardt, H., 1977. A synoppsis of the genera of Neotropical Carabidae (Insecta: Coleoptera). Quaestiones Entomologicae, 13: 346-493.

Rosa, T.A., M L.Y. Babata, C.M. Souza, D. Sousa, C.A. MelloPatiu, F.Z. Vaz-de-Mello \& J. Mendes, 2011. Arthropods associated with pig carrion in two vegetation profiles of Cerrado in the State of Minas Gerais, Brazil. Revista Brasileira de Entomologia, 55: 424-434.

Santos, W.E., 2012. Diversidade, sazonalidade e sucessão ecológica de Coleoptera (Insecta) associados ao processo de decomposição de Sus scrofa L. em Caatinga Paraibana. Dissertação (Mestrado em Ciências Biológicas: Zoologia) Universidade Federal da Paraíba. 70 p.

Smith, K.G.V., 1986. A manual of forensic entomolgy. London, British Museum, 205p.

Souza, A.M. \& A.X. Linhares, 1997. Diptera and Coleoptera of potential forensic importance in Southeastern Brazil: relative abundance and seasonality. Medical and Veterinary Entomology, 11: 8-12.

Ururahy-Rodrigues, A., J.A. Rafael, J.R. Pujol-Luz, A.L. Henriques, M.M.C. Queiroz, R.R. Barbosa \& M.N. Baroni, 2010. Association of Oxelytrum cayennense (Silphidae, Coleoptera) with Pig Carcasses (Sus scrofa, Suidae) in Terra Firme Areas in Manaus, Amazonas, Brazil. EntomoBrasilis, 3: $45-48$.

Vaz-de-Mello, F.Z., W.D. Edmonds, F.C. Ocampo \& P. Schoolmeesters, 2011. A multilingual key to the genera and 
subgenera of the subfamily Scarabaeinae of the New World (Coleoptera: Scarabaeidae). Zootaxa, 2854: 1-73.
Recebido em: 25/04/2012

Aceito em: 22/05/2102

Como citar este artigo:

Silva, R.C. \& W.E. Santos, 2012. Fauna de Coleoptera Associada a Carcaças de Coelhos Expostas em uma Área Urbana no Sul do Brasil. EntomoBrasilis, 5(3): 185-189.

Acessível em: http://www.periodico.ebras.bio.br/ojs/index.php/ebras/article/view/245
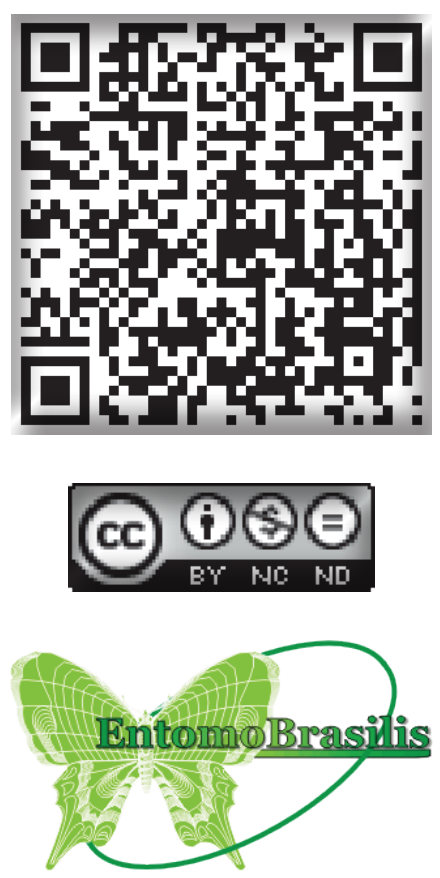